\title{
Anwärmen spart prandiales Insulin
}

Ein neues Gerät lässt mithilfe kontrollierter Erwärmung der Injektionsstelle prandiales Insulin rascher und effektiver wirken. So sinkt auch der Insulinverbrauch. Dies könnte für Typ-1- wie Typ-2-Diabetiker interessant sein.

„Schnell wirksames prandiales Insulin kommt der physiologischen Insulinfreisetzung nahe, ist aber noch nicht schnell genug. Wir müssen dafür sorgen, dass es noch rascher absorbiert werden und wirken kann" So umriss Elmar Schäfer, Geschäftsführer Deutschland des israelischen Unternehmens Insuline Medical, die Idee zur Entwicklung des InsuPad ${ }^{\mathrm{TM}}$.

Fenster auf - Piks - Fenster zu - Wärme Schäfer erläuterte Anwendung und Wirkprinzip des Hilfsmittels: „Es ist ein unauffälliges, etwa $2 \times 4 \mathrm{~cm}$ großes und 12 $\mathrm{g}$ schweres Gerät, wird tagsüber auf die Injektionsstelle geklebt, ist hautfreundlich und wasserfest. Zur Injektion muss sein Fenster aufgeklappt werden. Nach Wiederverschluss wird die Hautstelle automatisch auf $39,5^{\circ} \mathrm{C}$ erwärmt." Nach ca. 50 Minuten wird die Wärmezufuhr beendet. Dank der verbesserten Durchblutung werde das Insulin rascher ins Blut aufgenommen. „Abends wird der Pad abgenommen, die Kontrolleinheit aufgeladen und am nächsten Tag mit neuem Einweg-Fenster woanders befestigt", so Schäfer. Durch das tägliche Wechseln der Injektionsstelle wird zudem der Entstehung von Lipodystrophien entgegen gewirkt.

Prof. Dr. Andreas Pfützner vom Institut für Klinische Forschung und Entwicklung in Mainz ist auch Medizinischer Direktor Europa bei Insuline Medical. Er präsentierte eine klinische Studie zum neuen Gerät: „An 13 Zentren in ganz Deutschland wurden 145 ambulante Patienten mit intensivierter Insulintherapie - 90\% Typ-2-Diabetiker - in die Studie aufgenommen. Sie applizierten zu Beginn täglich mindestens 60 IE prandiales Analoginsulin." Das Prinzip funktioniert aber auch mit Humaninsulin.

Nach einer vierwöchigen Run-in-Phase zur Dosisoptimierung ihres Basalinsulins wurden die Teilnehmer offen auf Anwendung oder Nichtanwendung des neuen Geräts randomisiert. „Drei Monate später war der $\mathrm{HbA}_{1 c}$-Wert in den Gruppen mit vs. ohne das Pad von im Schnitt 7,2\% auf $6,2 \%$ bzw. $6,3 \%$ gesun- ken“, so Pfützner. „Die Patienten, die das neue Device anwendeten, benötigten aber im Mittel 19,4\% weniger prandiales Insulin als zu Studienbeginn, die in der Kontrollgruppe $8,1 \%$ mehr; der Unterschied betrug fast 28\%. "Hypoglykämien $(<63 \mathrm{mg} / \mathrm{dl}$ ) traten bei 3,3\% der Pad- und 6,2\% der Kontrollpatienten auf: „Sie waren in der Pad-Gruppe um relative 45\% verringert", so Pfützner. Beim Körpergewicht gab es eine Tendenz zugunsten der Pad-Patienten. „Die Therapiezufriedenheit war in beiden Gruppen ähnlich. Die meisten der Gerätetestgruppe wollten das Gerät nach der Studie behalten."

Eine Subgruppe von 32 Patienten in Mainz wurde mithilfe standardisierter Mahlzeiten genauer untersucht. Hier bestätigte sich, dass eine Dosis des postprandialen Insulins bei ähnlichen postprandialen Glukoseexkursionen wie zu Studienbeginn um $20-30 \%$ verringert werden konnte. „Mit dem neuen Gerät nutzt man das therapeutische Potenzial des Insulins besser“, so Pfützner. „Das Analoginsulin wird zum Turbo-Insulin.“ Die Studie wurde in Zusammenarbeit mit gen Glukosewerten verringert werden.

Veranstalter: Medtronic

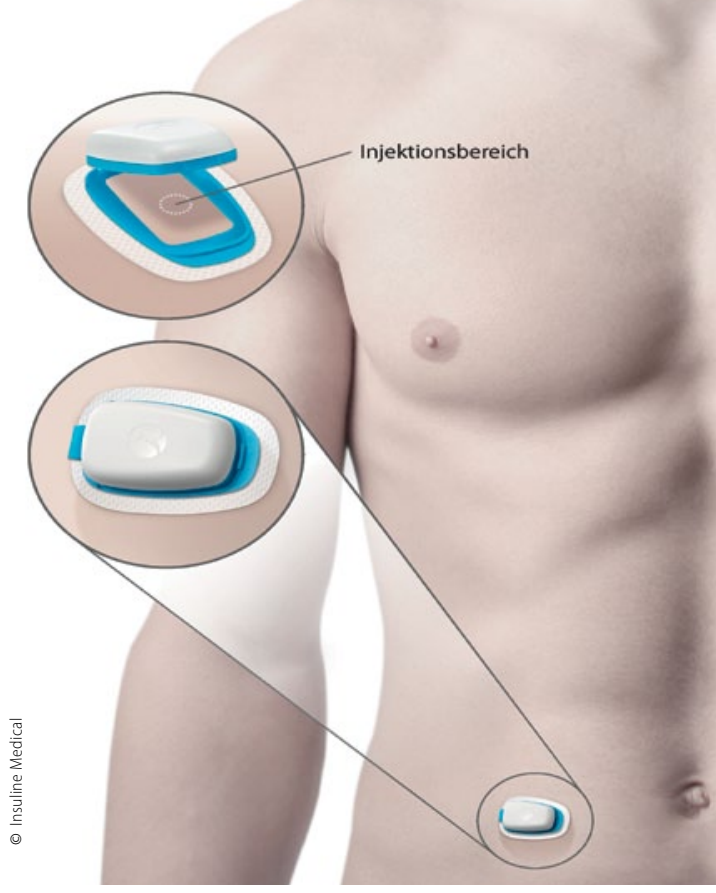

Das Erwärmungspad wird unauffällig auf die Haut geklebt und bleibt tagsüber dort.

der Barmer GEK durchgeführt und führte zur Anerkennung dieses Pads als erstattungsfähiges Hilfsmittel bei dieser Kasse. Die Anwendung ist mit 1,66 Euro täglich kalkuliert. „Wir hatten eine mindestens 10\%ige Insulineinsparung angestrebt, dies wurde weit übertroffen", so Pfützner. „Weitere Kostenträger haben signalisiert, dass sie sich der Barmer GEK anschließen werden, weil die Ergebnisse sie überzeugten." Das Pad soll als StarterSet und Monatspackung Ende 2013 auf den Markt kommen. Simone Reisdorf

Quelle: Pressekonferenz beim Diabeteskongress, 8.5.2013 in Leipzig, Veranstalter: Insuline Medical

\section{Sensorgestützte Insulinpumpentherapie entschärft Hypoglykämiegefahr}

Hypoglykämien können durch Insulinpumpen-Systeme mit zugeschaltetem kontinuierlichen Glukosemonitoring (CGM) und automatischer Insulinabschaltung bei niedri-

Bei schweren Hypoglykämien steigt die 5-Jahres-Mortalität um das 3,4-Fache signifikant im Vergleich zu Diabetikern mit milden oder ohne Hypoglykämien. Viele dieser Episoden werden aber von den Patienten nicht bemerkt, da sie im Schlaf auftreten, so Prof. Werner Kern aus Ulm. Eine online-Befragung von 1848 Diabetikern ergab, dass 28\% der Typ-1- und 11\% der Typ-2-Diabetiker 2012 eine oder mehrere schwere Hypoglykämien hatten, leichte gaben $94 \%$ bzw. $88 \%$ an. "Selbst nach milder Hypoglykämie ist der Blutzucker oft nur schwer wieder in den Griff zu bekommen", so Kern. Der genaue Glukoseverlauf lässt sich auch mit engmaschigen Messungen nicht abbilden, so Dr. Andreas Liebl, Bad Heilbrunn. BZ-Schwankungen seien mit einem erhöhten Hypoglykämierisiko assoziiert. CGM liefert einen kontinuierlichen Glukoseverlauf. So können Schwankungen und Hypoglykämien erkannt und vermieden werden. Typ-1-Diabetiker, die mind. 6 Tage/Woche ein CGM-Gerät einsetzen, können auch bei befriedigendem Ausgangs- $\mathrm{HbA}_{k}$ dessen signifikante Reduktion ohne $\mathrm{Zu}$ nahme des Hypoglykämierisikos erreichen. Das Insulinpumpensystem MiniMed Paradigm ${ }^{\circledast}$ $V_{e}{ }^{T M}$ erkennt Hypoglykämien und stoppt bei eingeschaltetem Glukosesensor die Insulinabgabe für zwei Stunden (low glucose suspend, LGS). Die Crossover-Studie ASPIRE* zeigte, dass die LGS-Funktion Dauer und Schwere von Hypoglykämien reduziert. Abdol A. Ameri

Quelle: Satellitensymposium beim Diabeteskongresses am 9.5.2013 in Leipzig, 